Paper:

\title{
Investigating Task Prioritization and Holistic Coordination Using Relative Jacobian for Combined 3-Arm Cooperating Parallel Manipulators
}

\author{
Rodrigo S. Jamisola Jr. and Frank Ayo Ibikunle \\ Electrical, Computer and Telecommunications Engineering Department, Botswana International University of Science and Technology (BIUST) \\ Private Bag 16, Palapye 10071, Botswana \\ E-mail: \{jamisolar, ibikunlef $\} @$ biust.ac.bw \\ [Received June 4, 2015; accepted September 11, 2015]
}

\begin{abstract}
A new modular relative Jacobian formulation for single end-effector control of combined 3-arm cooperating parallel manipulators is derived. It is based on a previous method of derivation for dual-arm robots, with the same approach of modularity and single endeffector control for combined manipulators. This paper will present this new formulation, as well as investigate task prioritization scheme to verify the claim that a single end-effector controller of combined manipulators will indeed implement a strict task prioritization, by intentionally adding more tasks. In addition, this paper will investigate a claim that the holistic approach to control of combined manipulators affords easier control coordination of each of the standalone components. Switching control from an individual manipulator control in the null space to relative control in the tasks space is shown to investigate the smoothness of task execution during switching. Simulation results using Gazebo 2.2.5 running in Ubuntu 14.04 is shown.
\end{abstract}

Keywords: Task prioritization, holistic control coordination, 3-arm cooperating parallel manipulators, single endeffector control, relative Jacobian, modular kinematics

\section{Introduction}

This work is geared towards a future goal of achieving a holistic control of combined manipulators, particularly, a humanoid that can perform more complicated motion such as performing a dive with somersault, jump and kick in the air, doing a cartwheel, etc. which are not possible at the current state of the art control for humanoids or for other combined manipulators such as quadrupeds and hexapods. Part of the challenge is on the complexity of the combined physical structures such that a holistic approach in its kinematics model and an accurate cancellation of its resulting dynamics require considerable effort.

This work is part of a series of studies to utilize modularity in the kinematics and dynamics expressions of the combined manipulators, expressed as a single manipula- tor (with single end-effector). In particular, this work considers 3-arm cooperating parallel manipulators controlled as a single manipulator. A modular kinematics expression is derived that is expressed in terms of the kinematics of each of the stand-alone manipulators. Of the single endeffector control of combined manipulators, its claims include: (1) strict implementation of task prioritization, and (2) a holistic approach to coordinated control. These two claims may prove to be crucial towards more complicated combined manipulators motions.

In this work, a modular relative Jacobian for the 3-arm cooperating parallel manipulator is derived. The concept of a relative Jacobian was first introduced in [1,2]. In a new derivation of a modular relative Jacobian for dualarms [3], a wrench transformation matrix was revealed that was not present or was not explicitly expressed in the previous relative Jacobians. Further studies shown in [4] highlighted the effects of the omission of the wrench transformation matrix on the exerted forces and moments at the dual-arm end-effectors, such that at certain configurations, its omission lead to non-contact for task that requires contact all the time. Asymmetric bimanual task was shown [5] for dual-arm performing at writing task using a relative Jacobian.

This work proposes to investigate more closely the task prioritization [6] and holistic coordinated control of combined 3-arm cooperating parallel manipulators (shown in Fig. 1) as a one single robot with a single end-effector. The two main reason behind this type of control includes: (1) a drastic increase the null-space dimension of the resulting combined manipulators and (2) the principles of single manipulator control can now be applied to the combined manipulators. In terms of the drastic control of the null-space dimension, consider a dual-arm robot with each arm having seven degrees-of-freedom (7DOFs). When each of the two arms is independently controlled in the full space, the resulting dual-arm robot has two degrees of redundancy (2-DORs). But when the arms are controlled in the relative full space, the resulting dualarm robot has 8-DORs.

Modularity on the proposed approach enhances ease of implementation of a single end-effector controller. Thus the resulting kinematics and dynamics expressions of the 


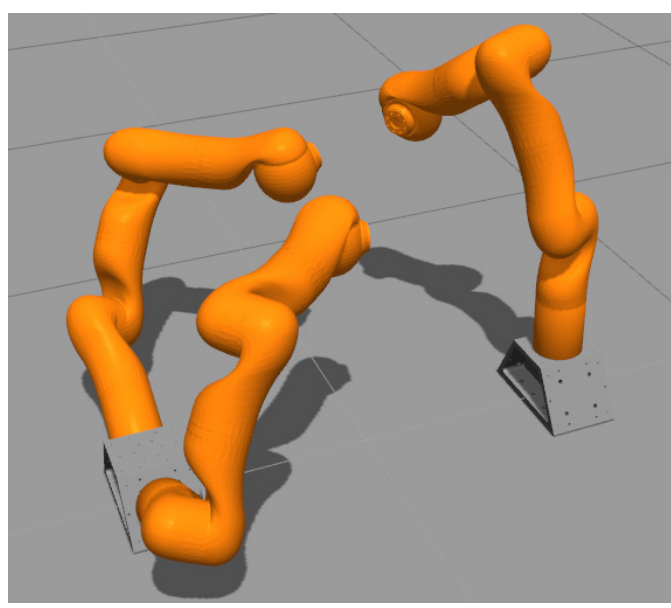

Fig. 1. A holistic coordinated motion of the 3-arm cooperating parallel manipulators. Simulation video is shown here: https://youtu.be/w87Ei7Z2Uis

resulting combined manipulators is derived based on the existing kinematics and dynamics of each of the standalone manipulators.

Most studies in cooperating manipulators are in dualarms [7-10], and in multi-arm cooperating manipulators [11-15]. To the best of our knowledge, this is the only existing study that considers exactly three arms in cooperating parallel manipulators. This number may not be crucial for cooperating manipulators, but it is crucial in the derivation of the single end-effector kinematics of combined manipulators. The increase of one more manipulator added to a dual-arm system defines a new relative Jacobian expression expressed in terms of the Jacobians of the stand-alone manipulators. This is a crucial step towards defining the relative Jacobian of four or more parallel manipulators cooperating together. The term "parallel" may have been a misnomer because this normally refers to manipulators with end-effectors that are rigidly connected to each together. However, we use the term "parallel" in this sense that the bases of the manipulators are rigidly connected to each other, as opposed to the "series" connection where a manipulator base is rigidly connected to the end-effector of another manipulator. Other manipulator kinematics study include $[16,17]$.

\section{Naming Convention for Symbols}

The naming convention for most symbols used in this work are shown in Table 1. Based on the schematic diagram of the 3-arm cooperating parallel manipulators in Fig. 2, the reference frames are assigned. The base reference frames are odd-numbered, while the end-effector reference frames are even-numbered. Relative position vectors connect the end-effectors.

Consider reference frames $\{i\}$ and $\{j\}$, such that ${ }^{i} p_{j}$ is the position of frame $\{j\}$ with respect to frame $\{i\}$, and ${ }^{i} R_{j}$ is the rotation of frame $\{j\}$ with respect to frame $\{i\}$. In addition, a Jacobian ${ }^{i} J_{j}$ can be expressed with respect
Table 1. Symbols-naming convention.

\begin{tabular}{|c|c|}
\hline Sym. & Description \\
\hline${ }^{i} p_{j}$ & $\begin{array}{l}\text { position of frame }\{j\} \text { w.r.t. frame }\{i\} \text {; its first } \\
\text { derivative is }{ }^{i} \dot{p}_{j}\end{array}$ \\
\hline${ }^{i} R_{j}$ & $\begin{array}{l}\text { orientation of }\{j\} \text { w.r.t. }\{i\} \text {; its first derivative } \\
\text { is }{ }^{i} \dot{R}_{j}\end{array}$ \\
\hline${ }^{i} \omega_{j}$ & rotational velocity of $\{j\}$ w.r.t. $\{i\}$ \\
\hline${ }^{i} J_{j}$ & {$\left[{ }^{i} J_{p j},{ }^{i} J_{o j}\right]^{T}$ Jacobian from $\{i\}$ to $\{j\}$} \\
\hline${ }^{i} J_{p j}$ & position component of Jacobian ${ }^{i} J_{j}$ \\
\hline${ }^{i} J_{o j}$ & orientation component of Jacobian ${ }^{i} J_{j}$ \\
\hline${ }^{1} J_{2}$ & Jacobian of $\operatorname{robot} A$ \\
\hline${ }^{3} J_{4}$ & Jacobian of robot $B$ \\
\hline${ }^{5} J_{6}$ & Jacobian of robot $C$ \\
\hline${ }^{2} J_{4}$ & relative Jacobian of dual-arm robots $A$ and $B$ \\
\hline${ }^{4} J_{6}$ & relative Jacobian of dual-arm robots $B$ and $C$ \\
\hline${ }^{2} J_{6}$ & relative Jacobian of dual-arm robots $A$ and $C$ \\
\hline$\frac{2}{3} J_{6}$ & relative Jacobian of 3-arm robots $A, B$ and $C$ \\
\hline$\dot{q}_{2}$ & joint velocities of robot $A$ \\
\hline$\dot{q}_{4}$ & locities of robot $B$ \\
\hline$\dot{q}_{6}$ & joint velocities of robot $C$ \\
\hline$\dot{q}_{24}$ & $\begin{array}{l}{\left[\dot{q}_{2}, \dot{q}_{4}\right]^{T} \text { joint velocities of dual-arm robots } A} \\
\text { and } B\end{array}$ \\
\hline$\dot{q}_{46}$ & $\begin{array}{l}{\left[\dot{q}_{4}, \dot{q}_{6}\right]^{T} \text { joint velocities of dual-arm robots } B} \\
\text { and } C\end{array}$ \\
\hline$\dot{q}_{246}$ & $\begin{array}{l}{\left[\dot{q}_{2}, \dot{q}_{4}, \dot{q}_{4}\right]^{T} \text { joint velocities of } 3 \text {-arm robots } A,} \\
B \text {, and } C\end{array}$ \\
\hline${ }_{3}^{2} p_{6}$ & 3 -arm relative position of $\{6\}$ w.r.t. $\{2\}$ \\
\hline${ }_{3}^{2} \dot{p}_{6}$ & $\begin{array}{l}\text { 3-arm relative translational velocity of }\{6\} \\
\text { w.r.t. }\{2\}\end{array}$ \\
\hline${ }_{3}^{2} \omega_{6}$ & $\begin{array}{l}\text { 3-arm relative rotational velocity of }\{6\} \text { w.r.t. } \\
\{2\}\end{array}$ \\
\hline
\end{tabular}

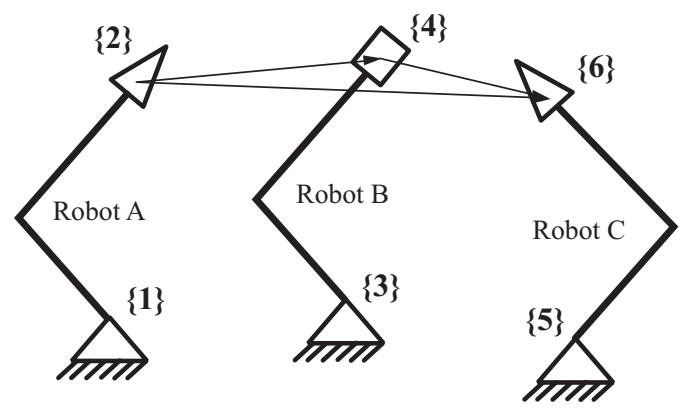

Fig. 2. An schematic diagram of a 3-arm cooperating parallel manipulator, with the corresponding reference frames and the relative position vectors.

to those frames. From the figure, we state the following conventions for the Jacobians of the standalone manipulators. The Jacobian for robot $A$ is ${ }^{1} J_{2}$, for robot $B$ is ${ }^{3} J_{4}$ and for robot $C$ is ${ }^{5} J_{6}$, each is expressed with respect to the indicated reference frame indices.

We assign the position Jacobian ${ }^{i} J_{p j}$ and orientation Jacobian ${ }^{i} J_{o j}$ as components of the Jacobian ${ }^{i} J_{j}$, that is, ${ }^{i} J_{j}=\left[{ }^{i} J_{p j},{ }^{i} J_{o j}\right]^{T}$. The joint velocities $\dot{q}_{i j}=\left[\dot{q}_{i}, \dot{q}_{j}\right]^{T}$, such $q_{i}$ and $q_{j}$ are the joint velocities of the robot with end-effector frames $\{i\}$ and $\{j\}$, respectively. For example ${ }^{1} J_{2}=\left[{ }^{1} J_{p 2},{ }^{1} J_{o 2}\right]^{T}$ is the Jacobian for $\operatorname{robot} A$, and ${ }^{2} J_{4}=\left[{ }^{2} J_{p 4},{ }^{2} J_{o 4}\right]^{T}$ is the relative Jacobian of the dual-arm 
consisting of robots $A$ and $B$. The dual-arm joint velocities $\dot{q}_{24}=\left[\dot{q}_{2}, \dot{q}_{4}\right]^{T}$, where $\dot{q}_{2}$ are the joint velocities of robot $A \dot{q}_{4}$ are the joint velocities of robot $B$.

\section{The Modular Relative Jacobian of 3-Arm Cooperating Parallel Manipulators}

Based on the frame assignment shown in Fig. 2, we present here the modular relative Jacobians for dual-arms as derived in [3]. The relative Jacobian for a dual-arm consisting of robots $A$ and $B$ is

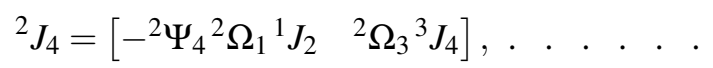

the relative Jacobian of a dual-arm consisting of robots $B$ and $C$ is

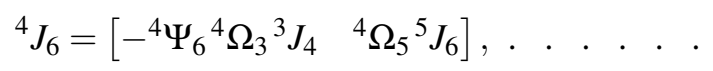

and lastly, the relative Jacobian for dual-arm robots $A$ and $C$ is

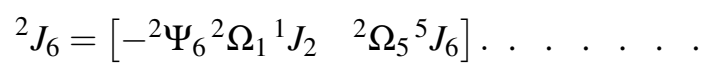

Such that the wrench transformation matrix ${ }^{i} \Psi_{j}$ is defined as

$$
{ }^{i} \Psi_{j}=\left[\begin{array}{cc}
I & -S\left({ }^{i} p_{j}\right) \\
0 & I
\end{array}\right]
$$

and the rotation matrix ${ }^{i} \Omega_{j}$ is expressed as

$$
{ }^{i} \Omega_{j}=\left[\begin{array}{cc}
{ }^{i} R_{j} & 0 \\
0 & { }^{i} R_{j}
\end{array}\right] .
$$

Given $\omega=\left[\omega_{x}, \omega_{y}, \omega_{z}\right]^{T}$, the operator $S(\omega)$ is the skew symmetric operator used to replace the cross-product operator and is expressed as

$$
S(\omega)=\left[\begin{array}{ccc}
0 & -\omega_{z} & \omega_{y} \\
\omega_{z} & 0 & -\omega_{x} \\
-\omega_{y} & \omega_{x} & 0
\end{array}\right]
$$

To complete the definition of the modular dual-arm manipulators the shown robots in Fig. 2, we define the relative position vectors between the end-effectors, called ${ }^{i} p_{j}$ for the paired robots. We express them here as

$$
\begin{aligned}
{ }^{2} p_{4} & ={ }^{2} R_{1}\left({ }^{1} p_{3}+{ }^{1} R_{3}{ }^{3} p_{4}-{ }^{1} p_{2}\right) \\
{ }^{4} p_{6} & ={ }^{4} R_{3}\left({ }^{3} p_{5}+{ }^{3} R_{5}{ }^{5} p_{6}-{ }^{3} p_{4}\right) \\
{ }^{2} p_{6} & ={ }^{2} R_{1}\left({ }^{1} p_{5}+{ }^{1} R_{5}{ }^{5} p_{6}-{ }^{1} p_{2}\right) .
\end{aligned}
$$

To derive the modular relative Jacobian for the 3arm cooperating parallel manipulators, we invoke the approach used in [3], that is, we express translational and rotational velocities of the end-effectors with respect to each other. Thus the relative position of frame $\{6\}$ with respect to frame $\{2\}$ is expressed as

$$
{ }_{3}^{2} p_{6}={ }^{2} p_{4}+{ }^{2} R_{4}{ }^{4} p_{6} .
$$

We take the derivative of the above equation to get

$$
\begin{aligned}
{ }_{3}^{2} \dot{p}_{6} & ={ }^{2} \dot{p}_{4}+{ }^{2} \dot{R}_{4}{ }^{4} p_{6}+{ }^{2} R_{4}{ }^{4} \dot{p}_{6} \\
& ={ }^{2} \dot{p}_{4}+S\left({ }^{2} \omega_{4}\right){ }^{2} R_{4}{ }^{4} p_{6}+{ }^{2} R_{4}{ }^{4} \dot{p}_{6} \\
& ={ }^{2} \dot{p}_{4}-S\left({ }^{2} R_{4}{ }^{4} p_{6}\right){ }^{2} \omega_{4}+{ }^{2} R_{4}{ }^{4} \dot{p}_{6} .
\end{aligned}
$$

The linearity of angular velocities allows us the express the relative angular velocity of frame $\{6\}$ with respect to frame $\{2\}$ as

$$
{ }_{3}^{2} \omega_{6}={ }^{2} \omega_{4}+{ }^{2} R_{4}{ }^{4} \omega_{6} .
$$

By combining Eqs. (9) and (10), we get

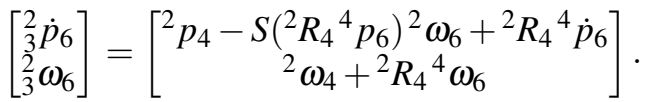

We then simplify the above expression by combining linear and rotational terms together and express the result in terms of the dual-arm relative Jacobians shown from Eqs. (1) to (3) to get

$$
\begin{aligned}
& {\left[\begin{array}{l}
{ }_{3}^{2} \dot{p}_{6} \\
2 \\
3
\end{array} \omega_{6}\right]} \\
& =\left[\begin{array}{c}
{ }^{2} J_{p 4} \dot{q}_{24}-S\left({ }^{2} R_{4}{ }^{4} p_{6}\right){ }^{2} J_{o 4} \dot{q}_{24}+{ }^{2} R_{4}{ }^{4} J_{p 6} \dot{q}_{46} \\
{ }^{2} J_{o 4} \dot{q}_{24}+{ }^{2} R_{4}{ }^{4} J_{o 6} \dot{q}_{46}
\end{array}\right] \\
& =\left[\left[\begin{array}{cc}
I & -S\left({ }^{2} R_{4}{ }^{4} p_{6}\right) \\
0 & I
\end{array}\right]\left[\begin{array}{c}
{ }^{2} J_{p 4} \\
{ }^{2} J_{o 4}
\end{array}\right] \dot{q}_{24} \ldots\right. \\
& \left.\ldots+\left[\begin{array}{cc}
{ }^{R} R_{4} & 0 \\
0 & { }^{2} R_{4}
\end{array}\right]\left[\begin{array}{c}
{ }^{4} J_{p 6} \\
{ }^{4} J_{o 6}
\end{array}\right] \dot{q}_{46}\right] \\
& =\left[\left[\begin{array}{cc}
I & -S\left({ }^{2} R_{4}{ }^{4} p_{6}\right) \\
0 & I
\end{array}\right]{ }^{2} J_{4}\left[\begin{array}{cc}
{ }^{2} R_{4} & 0 \\
0 & { }^{2} R_{4}
\end{array}\right]{ }^{4} J_{6}\right]\left[\begin{array}{l}
\dot{q}_{24} \\
\dot{q}_{46}
\end{array}\right] \\
& =\left[\begin{array}{ll}
2,4 \Psi_{6}{ }^{2} J_{4} & 2 \Omega_{4}{ }^{4} J_{6}
\end{array}\right]\left[\begin{array}{l}
\dot{q}_{24} \\
\dot{q}_{46}
\end{array}\right] \\
& =\left[{ }^{2,4} \Psi_{6}\left[-{ }^{2} \Psi_{4}{ }^{2} \Omega_{1}{ }^{1} J_{2} \quad{ }^{2} \Omega_{3}{ }^{3} J_{4}\right] \ldots\right. \\
& \ldots{ }^{2} \Omega_{4}\left[\begin{array}{ll}
-{ }^{4} \Psi_{6}{ }^{4} \Omega_{3}{ }^{3} J_{4} & { }^{4} \Omega_{5}{ }^{5} J_{6}
\end{array}\right]\left[\begin{array}{l}
\dot{q}_{24} \\
\dot{q}_{46}
\end{array}\right] \\
& =\left[-{ }^{2,4} \Psi_{6}{ }^{2} \Psi_{4}{ }^{2} \Omega_{1}{ }^{1} J_{2}\left({ }^{2,4} \Psi_{6}{ }^{2} \Omega_{3}-{ }^{2} \Omega_{4}{ }^{4} \Psi_{6}{ }^{4} \Omega_{3}\right){ }^{3} J_{4} \ldots\right. \\
& \left.\ldots{ }^{2} \Omega_{4}{ }^{4} \Omega_{5}{ }^{5} J_{6}\right]\left[\begin{array}{l}
\dot{q}_{24} \\
\dot{q}_{46}
\end{array}\right] \text {, }
\end{aligned}
$$

where ${ }^{i, j} \Psi_{k}$ means that the wrench transformation matrix has the cross-product operator defined as $S\left({ }^{i} R_{j}{ }^{j} p_{k}\right)$. In the second to the last equality of Eq. (12), we substitute the dual-arm relative Jacobians of Eqs. (1) and (2). To further simplify, we group terms together, such that the modular relative Jacobian for a 3-arm cooperating parallel manipulator can be expressed as

$$
\begin{array}{r}
{ }_{3}^{2} J_{6} \\
=\left[-{ }^{2,4} \Psi_{6}{ }^{2} \Psi_{4}{ }^{2} \Omega_{1}{ }^{1} J_{2}\left({ }^{2,4} \Psi_{6}{ }^{2} \Omega_{3}-{ }^{2} \Omega_{4}{ }^{4} \Psi_{6}{ }^{4} \Omega_{3}\right)^{3} J_{4} \ldots\right. \\
\left.\ldots{ }^{2} \Omega_{4}{ }^{4} \Omega_{5}{ }^{5} J_{6}\right] .
\end{array}
$$

Simplification of terms are shown in [18]. Upon simplification, the relative Jacobian of the 3-arm cooperating parallel manipulator becomes

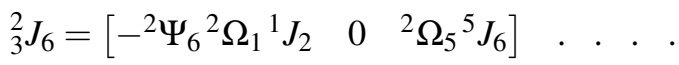


which is identical to Eq. (3), except for the middle zero column.

Comparing Eq. (14) to Eq. (3) it would seem that we have not gained enough in terms of expressing the relative Jacobian of the 3-arm cooperating parallel manipulators. However, this new formulation is in fact a consequence of the method of formulation based on paired-arm manipulation. This approach is commonly found in nature $[19,20]$. Thus, the third arm will always move in the null-space of the dual arm. A holistic modular kinematic expression for the 3-arm cooperating parallel manipulator can be expressed as

$$
\begin{array}{r}
\dot{q}_{246}={ }_{3}^{2} J_{6}^{+2} \dot{x}_{6}+\left(I-{ }_{3}^{2} J_{6}^{+2}{ }_{3} J_{6}\right){ }_{3}^{2} J_{4}^{+2} \dot{x}_{4} \ldots \\
\ldots+\left(I-\frac{2}{3} J_{6}^{+2} \frac{2}{3} J_{6}\right)\left(I-\frac{2}{3} J_{4}^{+2} J_{3} J_{4}\right) \nabla z
\end{array}
$$

where $\dot{q}_{246}=\left[\dot{q}_{2}, \dot{q}_{4}, \dot{q}_{6}\right]^{T},{ }_{3}^{2} J_{4}=\left[{ }^{2} J_{4} 0\right],{ }^{2} \dot{x}_{6}=\left[{ }^{2} \dot{p}_{6},{ }^{2} \omega_{6}\right]^{T}$, ${ }^{2} \dot{x}_{4}=\left[{ }^{2} \dot{p}_{4},{ }^{2} \omega_{4}\right]^{T}$, and $\nabla z$ is the null space posture.

The expression in Eq. (15) shows modularity in expressing the complete kinematics of the 3-arm cooperating parallel manipulators in both task space and nullspace velocities. The null space projection of $\nabla z$ can be computed as shown in [6], where maximum number of tasks was utilized and prioritized despite singularities.

\section{Simulation Using Gazebo}

The section shows the results using Gazebo simulator. The controller in the simulation is a controller with purely kinematic information, without any dynamics information included. This can be a limitation in the simulation. The velocity controller is expressed as

$$
\begin{aligned}
\dot{q}_{246} & =J_{R}^{+} \Delta\left(x_{R}\right)+\left(I-J_{R}^{+} J_{R}\right){ }_{3}^{1} J_{2}^{+} \Delta\left({ }^{1} x_{2}\right) \\
& \ldots+\left(I-J_{R}^{+} J_{R}\right)\left(I-{ }_{3}^{1} J_{2}^{+1}{ }_{3} J_{2}\right){ }_{3}^{2} J_{4}^{+} \Delta\left({ }^{2} x_{4}\right) \ldots \\
& \ldots+\left(I-J_{R}^{+} J_{R}\right)\left(I-{ }_{3} J_{2}^{+1}{ }_{3} J_{2}\right)\left(I-{ }_{3} J_{4}^{+2} J_{3}\right) \nabla z
\end{aligned}
$$

where $J_{R}={ }_{3}^{2} J_{6}$ and $x_{R}={ }_{3}^{2} x_{6}$ is the relative position and orientation vector. For the delta function, given $x$ as the input,

$$
\Delta(x)=k_{P}\left(x_{d}-x\right)+k_{V}\left(\dot{x}_{d}-\dot{x}\right)+k_{I} \sum_{t=0}\left(x_{d}-x\right)
$$

where $x_{d}$ is the desired $x, \dot{x}_{d}$ desired velocity of $x_{d}, \dot{x}$ is the velocity of $x, t$ is the time, and $k_{p}, k_{v}$, and $k_{i}$ are the proportional, derivative, and integral gains. The 3 -arm nullspace Jacobians are ${ }_{3}^{2} J_{4}=\left[\begin{array}{ll}{ }^{2} J_{4} & 0\end{array}\right]$ and ${ }_{3}^{1} J_{2}=\left[\begin{array}{lll}{ }^{1} J_{2} & 0 & 0\end{array}\right]$. The $\nabla z$ is the null-space gradient that controls the posture of the arms, such that $\nabla(z)=\left[\Delta\left(q_{2}\right), \Delta\left(q_{4}\right), \Delta\left(q_{6}\right)\right]^{T}$.

The desired values are the following (with lengths in meters and angles in degrees): ${ }_{3}^{2} x_{6 d}=[0,0,0.3,0,180,0]^{T} \quad(x, \quad y, \quad$ and $z$ position and roll, pitch and yaw orientation), ${ }^{2} x_{4 d}=$ $[-0.3,0,0,0,0,0]^{T}, \quad q_{2 d}=[0,+60,0,-45,0,-45,0]^{T}$, $q_{4 d}=[0,-60,0,+45,0,+45,0]^{T}, \quad$ and $q_{6 d}=[0,+60,0,-45,0,-45,0]^{T}$. All desired velocities are zero. The desired values ${ }^{1} x_{2 d}$ changes according in a point-to-point motion to the time increment

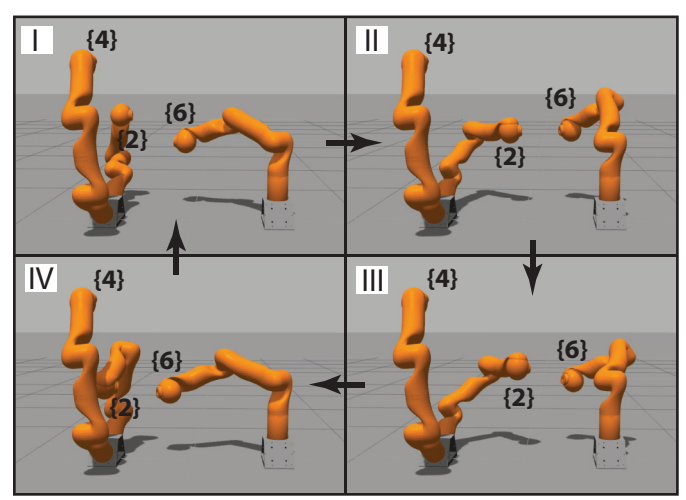

Fig. 3. Snapshots when robot $B$ (with frame $\{4\}$ endeffector) of the 3 -arm cooperating parallel manipulators is not moving.

of $1 \quad s$ as follows: ${ }^{1} x_{2 d}=[0.5,0,0.5,90,0,0]_{t=0}^{T}$, ${ }^{1} x_{2 d}=[0.5,-0.5,0.5,90,0,0]_{t=1}^{T}, \quad{ }^{1} x_{2 d}=$ $[0,-0.5,0.5,90,0,0]_{t=2}^{T}$, and ${ }^{1} x_{2 d}=[0,0,0.5,90,0,0]_{t=3}^{T}$. Then ${ }^{1} x_{2 d}$ loops back in a $4 s$ cycle of desired values.

Note that Gazebo simulator does not run in realtime. The gains are set at $k_{P}=3000, k_{V}=200$, and $k_{I}=0.1$. Note that the $\Delta(q)$ function in the null-space used $k_{P}=200$, and $k_{V}=k_{I}=0$.

Three sets of simulation experiments are shown here: (1) when robot $B$ is stationary such that $\{6\}$ moves w.r.t. to $\{2\}$ while $\{4\}$ is not moving (as shown in Fig. 3 with performance errors shown in Fig. 4), (2) all three robots end-effectors are moving in coordinated motion (as shown in Fig. 5 with performance errors shown in Fig. 6), and (3) robot $B$ goes in and out of coordinated motion while $\{6\}$ uninterruptedly moves w.r.t. $\{2\}$ (as shown in Fig. 7 with numerical errors shown in Fig. 8).

\section{Conclusion}

Performance errors shown from Figs. 4 to 8 showed consistent results such that the motion of robot $B$, being stationary or moving in a holistic coordinated motion, does not affect the relative motion between robots $A$ and $C$. Thus the shown identical errors in columns one and two of Figs. 4, 6, and 8, while the errors in column three of the same figures vary. This kind of strict task prioritization results is consistent with a single end-effector controller.

\section{References:}

[1] C. Lewis and A. Maciejewski, "Trajectory generation for cooperating robots," IEEE Int. Conf. on Systems Engineering 1990, pp. 300303, 1990 .

[2] C. Lewis, "Trajectory generation for two robots cooperating to perform a task," Proc. of IEEE Int. Conf. on Robotics and Automation 1996, Vol.2, pp. 1626-1631, Apr. 1996.

[3] R. S. Jamisola and R. G. Roberts, "A more compact expression of relative jacobian based on individual manipulator jacobians," Robotics and Autonomous Systems, Vol.63, pp. 158-164, 2015.

[4] R. S. Jamisola Jr., P. Kormushev, D. G. Caldwell, and F. Ibikunle, "Modular relative jacobian for dual-arms and the wrench transformation matrix," 2015 IEEE 7th Int. Conf. on Cybernetics and Intel- 


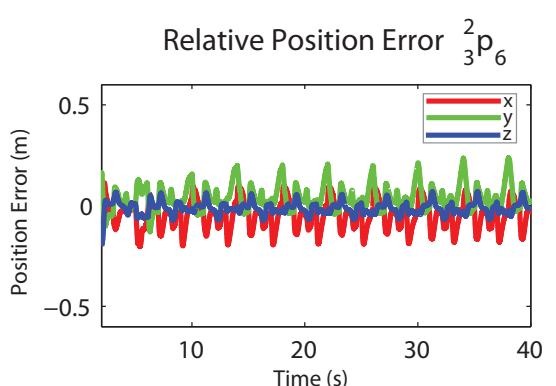

Relative Orientation Error ${ }_{3}^{2} \omega_{6}$
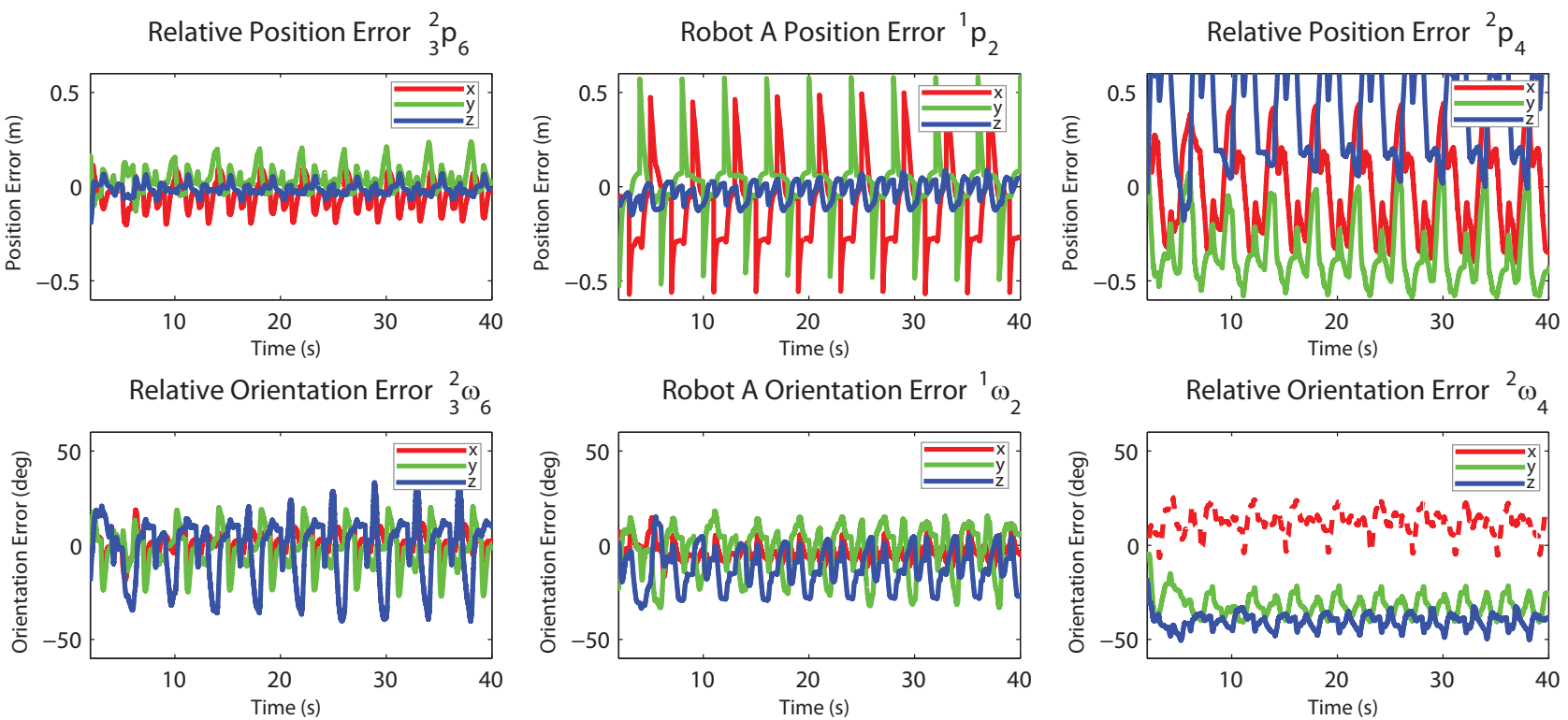

Robot A Orientation Error ${ }^{1} \omega_{2}$
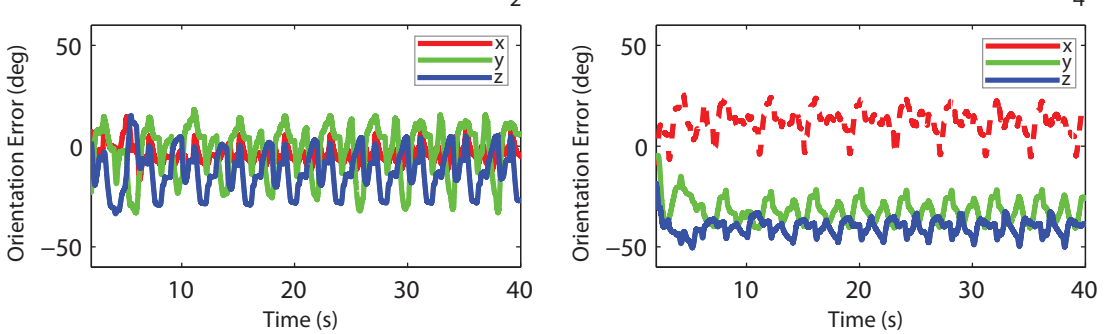

Fig. 4. Position and orientation errors when the robot $B$ is not moving. The first column of position and orientation errors are from the relative motion of frame $\{6\}$ w.r.t frame $\{2\}$; the second column are the errors of robot $A$ end-effector motion w.r.t to its base; and the third column are the errors for the relative motion of frame $\{4\}$ w.r.t. frame $\{2\}$. In this case the position errors are large because robot $B$ is not moving, but orientation error is not that large because the desired relative orientation remains constant.

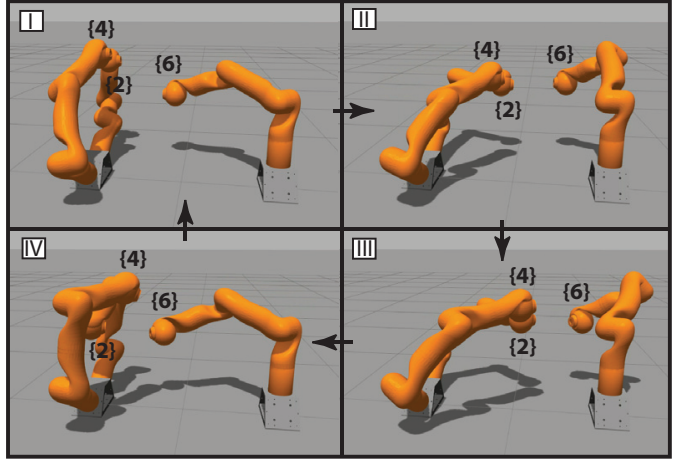

Fig. 5. Snapshots when all the robots are moving.

ligent Systems (CIS) and Robotics, Automation and Mechatronics (RAM), pp. 181-186, July 15-17, 2015.

[5] J. Lee, P. Chang, and R. S. Jamisola, "Relative impedance control for dual-arm robots performing asymmetric bimanual tasks," IEEE Trans. on Industrial Electronics, Vol.61, No.7, pp. 3786-3796, 2014.

[6] R. S. Jamisola, P. H. Chang, and J. Lee, "Guaranteeing task prioritization for redundant robots given maximum number of tasks and singularities," 2012 IEEE Region 10 Conf., TENCON2012, pp. 1-6, 2012.

[7] A. Tavasoli, M. Eghtesad, and H. Jafarian, "Two-time scale control and observer design for trajectory tracking of two cooperating robot manipulators moving a flexible beam," Robotics and Autonomous Systems, Vol.57, No.2, pp. 212-221, 2009.

[8] F. Caccavale, P. Chiacchio, A. Marino, and L. Villani, "Sixdof impedance control of dual-arm cooperative manipulators," IEEE/ASME Trans. on Mechatronics, Vol.13, No.5, pp. 576-586, 2008.

[9] C. Smith, Y. Karayiannidis, L. Nalpantidis, X. Gratal, P. Qi, D. V. Dimarogonas, and D. Kragic, "Dual arm manipulation - a survey," Robotics and Autonomous Systems, 2012.

[10] T. Wimböck and C. Ott, "Dual-arm manipulation," Towards Service Robots for Everyday Environments, pp. 353-366, Springer, 2012.

[11] S. A. A. Moosavian and R. Rastegari, "Multiple-arm space freeflying robots for manipulating objects with force tracking restrictions," Robotics and Autonomous Systems, Vol.54, No.10, pp. 779-788, 2006.
[12] M. D. Zivanovic and M. Vukobratovic, "Multi-arm cooperating robots: dynamics and control," Springer Science \& Business Media, Vol.30, 2006.

[13] W. Gueaieb, F. Karray, and S. Al-Sharhan, "A robust hybrid intelligent position/force control scheme for cooperative manipulators," IEEE/ASME Trans. on Mechatronics, Vol.12, No.2, pp. 109-125, 2007.

[14] F. Caccavale and M. Uchiyama, "Cooperative manipulators," Springer Handbook of Robotics, pp. 701-718, Springer, 2008.

[15] V. Panwar, N. Kumar, N. Sukavanam, and J.-H. Borm, "Adaptive neural controller for cooperative multiple robot manipulator system manipulating a single rigid object," Applied Soft Computing, Vol.12, No.1, pp. 216-227, 2012.

[16] R. S. Jamisola, A. A. Maciejewski, and R. G. Roberts, "Failuretolerant path planning for kinematically redundant manipulators anticipating locked-joint failures," IEEE Trans. on Robotics, Vol.22, No.4, pp. 603-612, 2006.

[17] R. S. Jamisola, "Optimization of failure-tolerant workspaces for redundant manipulators," Philippine Science Letters, Vol.3, Issue 1, pp. 66-75, 2010.

[18] R. S. Jamisola, C. Mastalli, and I. Frank, "Modular relative jacobian for combined 3-arm parallel manipulators," Int. J. of Mechanical Engineering and Robotics Research, to be appeared, 2015.

[19] R. W. Byrne, J. M. Byrne, et al., "Manual dexterity in the gorilla: bimanual and digit role differentiation in a natural task," Animal Cognition, Vol.4, No.3-4, pp. 347-361, 2001.

[20] D. A. Rosenbaum, K. M. Chapman, M. Weigelt, D. J. Weiss, and R. van der Wel, "Cognition, action, and object manipulation," Psychological Bulletin, Vol.138, No.5, p. 924, 2012. 

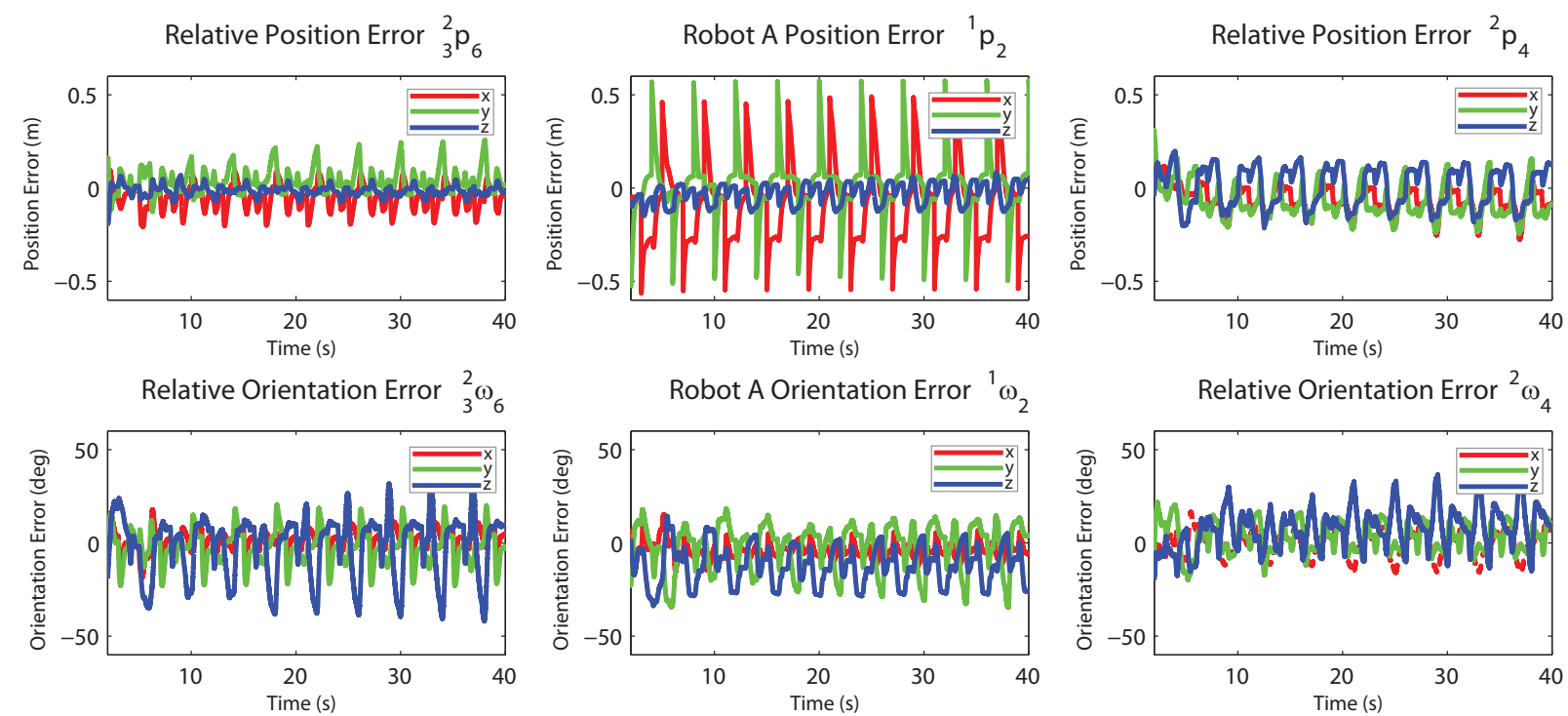

Fig. 6. The position and orientation errors as in Fig. 4, but with all the robots moving.

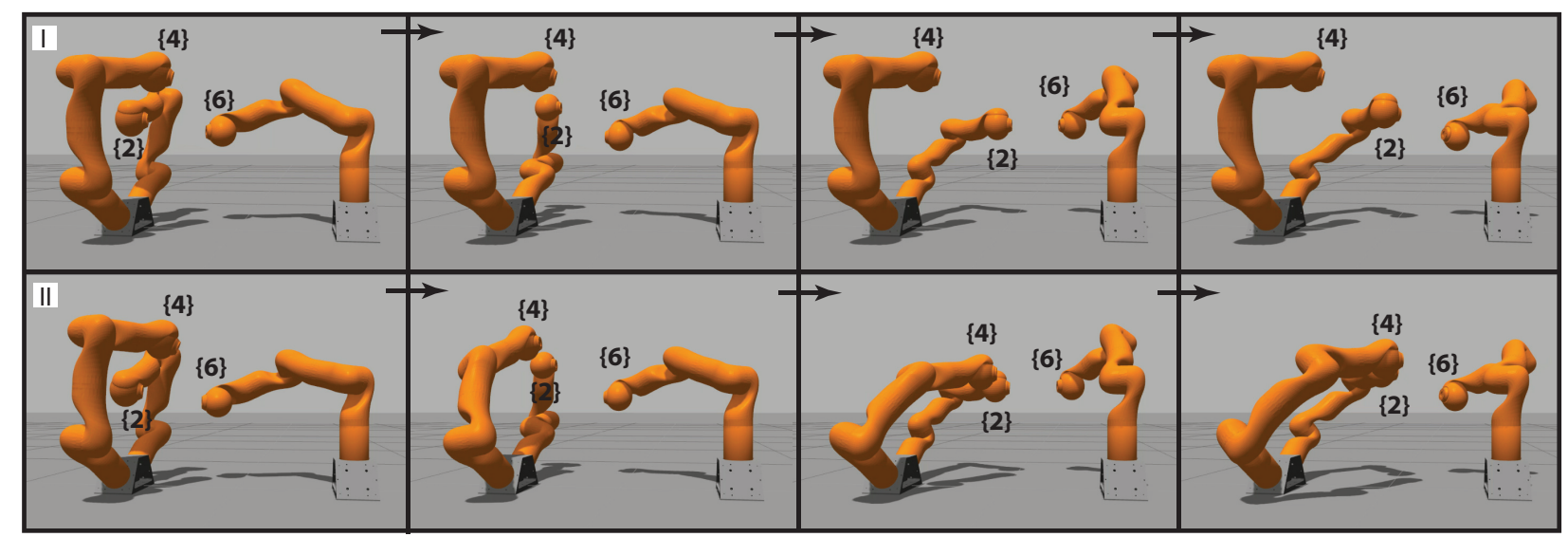

Fig. 7. Snapshots of 3 -arm cooperating parallel manipulator alternates from photo strip I (when robot $B$ with frame $\{4\}$ end-effector is not moving) to photo strip II (when all the robots are moving).

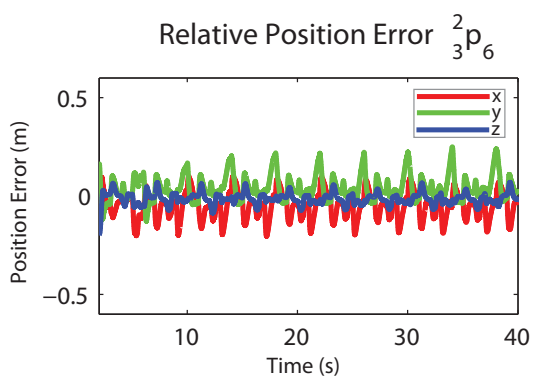

Relative Orientation Error ${ }_{3}^{2} \omega_{6}$

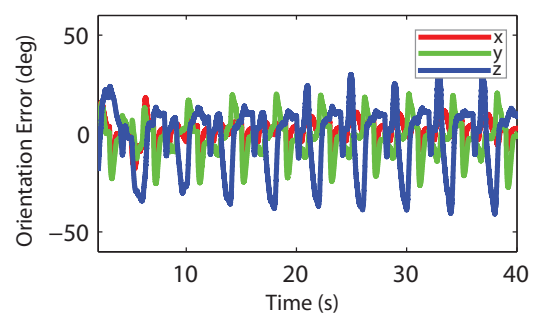

Robot A Position Error ${ }^{1} \mathrm{p}_{2}$

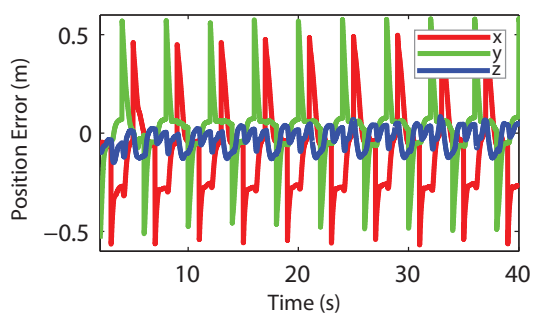

Robot A Orientation Error ${ }^{1} \omega_{2}$

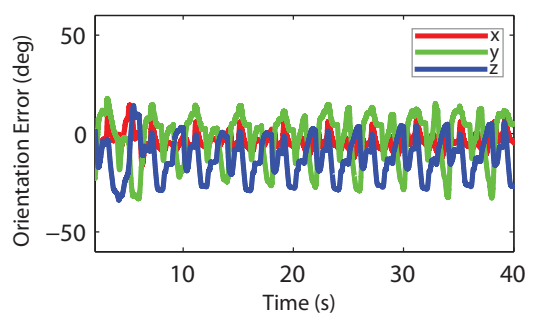

Relative Position Error ${ }^{2} \mathrm{p}_{4}$

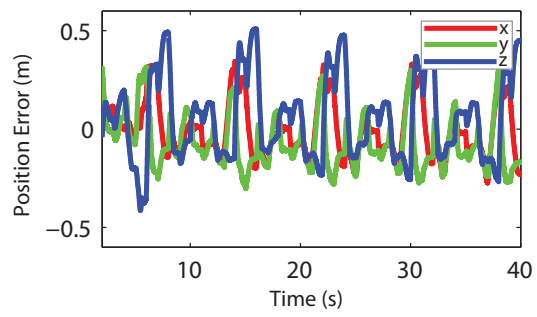

Relative Orientation Error ${ }^{2} \omega_{4}$

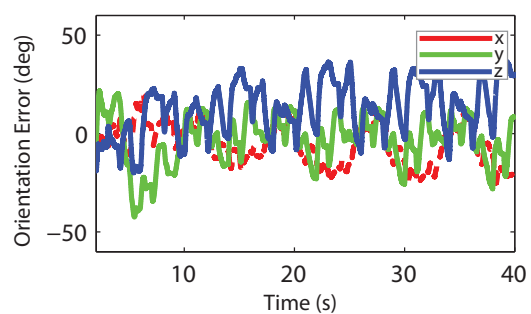

Fig. 8. Position and orientation errors as in Figs. 4 and $\mathbf{6}$ when robot $B$ alternates from moving with the rest of the robots to being stationary. 


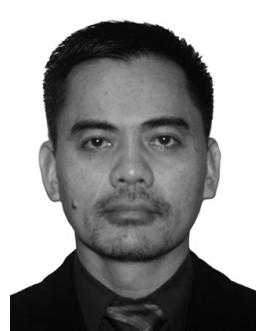

Name:

Rodrigo S. Jamisola, Jr.

\section{Affiliation:}

Senior Lecurer, Department of Electrical, Computer and Telecommunications Engineering Department, Botswana International University of Science and Technology (BIUST)

\section{Address:}

Private Bag 16, Palapye 10071, Botswana

Brief Biographical History:

1993 Received his B.S. degree in Mechanical Engineering from the University of the Philippines-Diliman

2001 Received his M.E. degree (research-based) in Mechanical

Engineering from the National University of Singapore

2006 Received his M.Sc. degree in Electrical and Computer Engineering from Colorado State University

2009 Received his Ph.D. degree in Electronics and Communications

Engineering from De La Salle University-Manila

2008- Assistant Professor, De La Salle University

2011- R\&D Manager, Toyota Motor Philippines

2011-2013 Post-doctoral Research Fellow at Daegu-Gyeongbuk Institute of Science and Technology in South Korea

2013-2014 Istituto Italiano di Tecnologia in Genova, Italy

2015- Senior Lecturer at Botswana International University of Science and

Technology

Main Works:

- His research interest includes control of combined manipulators,

machine learning, numerical optimization, and human-machine interfaces.

Membership in Academic Societies:

- The Institute of Elctrical and Electronics Engineers (IEEE)

- The American Society of Mechanical Engineers (ASME)

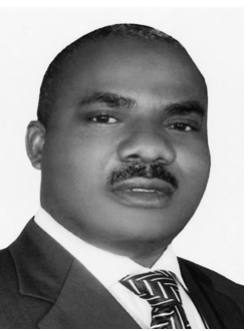

Name:

Frank Ayo Ibikunle

\section{Affiliation:}

Professor, Telecommunications Engineering and the Head of Department of Electrical, Computer and Telecommunications Engineering Department, Botswana International University of Science and Technology (BIUST)

Address:

Private Bag 16, Palapye 10071, Botswana

Brief Biographical History:

1986/1997 Received his first degree in Electrical/Electronic Engineering and won a Scholarship award to study for his Masters and Ph.D. degrees in Information and Telecommunications Engineering

2007-2013 Electrical \& Information Engineering Department, Covenant University

2013 Botswana International University of Science and Technology

Main Works:

- His areas of research are in Mobile and Broadband Wireless Access

Technologies (i.e. OFDMA, MIMO, Satellite, WiFi/WiMAX \&

2G/3G/LTE); Next Generation Converged Networks; Communication

Network and Security; Cognitive radio, Ad-hoc and Sensor networks;

Artificial Intelligence concepts (i.e., Neural Networks, Expert systems \&

Fuzzy logics); Cloud Computing and its application; and Radio Channel

Propagation \& Modelling in Wireless communications.

Membership in Academic Societies:

- Council for the Regulation of Engineering in Nigeria (COREN)

- Nigerian Society of Engineers (NSE)

- The Institute of Electrical and Electronics Engineers (IEEE)

- Nigerian Institute of Management (NIM) 